\title{
DIMENSIONES CONCEPTUALES PARA EVALUAR LA EFECTIVIDAD DE LA EVALUACIÓN DEL IMPACTO AMBIENTAL, BAJO LA LEGISLACIÓN AMBIENTAL PERUANA
}

Diego Espinoza Ortiz ORCID: 0000-0002-3684-7401

Gaspar Jiménez Peña

ORCID: 0000-0002-6390-1098

José Páez Warton

ORCID: 0000-0001-8345-0493

Centro de Altos Estudios Nacionales, Escuela de Posgrado

Diego Evangelista Vargas ORCID: 0000-0002-9017-5183

Milward Salas Delgado ORCID: 0000-0002-5631-1436

Marta Ramos Guillén ORCID: 0000-0002-4494-5562

Henrry Carrera Saavedra ORCID: 0000-0002-6507-2553

María Ángeles Natividad ORCID: 0000-0002-9736-1114

Universidad Nacional Mayor de San Marcos

Resumen: A treinta años de su implantación en la legislación nacional del Perú, la Evaluación del Impacto Ambiental (EIA) ha sido revisada con la finalidad de determinar qué indicadores podrían emplearse para evaluar su efectividad. Fueron analizadas fuentes de información sobre la calidad y efectividad de la EIA para el uso sostenible de recursos naturales, logrando un modelo conceptual de cadena de valor para la gestión por resultados desde la administración pública, identificando los insumos y las 
actividades para ofrecer un servicio de certificación ambiental que resulte específicamente en el uso sostenible de recursos naturales, y contribuya al goce de un ambiente equilibrado y adecuado al desarrollo de la vida. De la revisión efectuada a los trabajos de Chanchitpricha y Bond (2013), Bond et al. (2015) y Pope et al. (2018), entre otros, se distinguen seis dimensiones que permiten evaluar la efectividad de la EIA, referido al uso sostenible de los recursos naturales, que son: i) normativa, alcanzando las metas establecidas en las políticas; ii) participativa, involucrando de forma segura y confiable a las partes interesadas; iii) organizacional, facilitando la gestión del conocimiento sobre la EIA como herramienta de la gestión ambiental; iv) transaccional, gestionando de forma eficiente los recursos en la ejecución de la EIA, como tiempo, financieros, alcance o requisitos y habilidades del personal; v) procedimental, debido a un marco procedimental coherente y fiable; y vi) sustantiva, debido a la eficacia de la decisión tomada por la autoridad competente respecto de la viabilidad ambiental del proyecto sometido a la EIA. El trabajo es una contribución a mayores estudios sobre la efectividad de la EIA en el abordaje de los problemas ambientales y sociales que actualmente enfrentamos, y las posibles tendencias a presentarse en las futuras décadas.

Palabras clave: Evaluación del impacto ambiental, Sostenibilidad, Efectividad, Gestión por resultados, Gestión pública, Perú.

\title{
Conceptual Dimensions to Evaluate the Effectiveness of the Environmental Impact Assessment, under Peruvian Environmental Legislation
}

\begin{abstract}
: 30 years after its implementation in the national legislation of Peru, the Environmental Impact Assessment (EIA) has been revised in order to determine which indicators could be used to evaluate its effectiveness. Sources of information on the quality and effectiveness of the EIA for the sustainable use of natural resources were analyzed, achieving a conceptual model of a value chain for results-based management from the public administration, identifying the inputs and activities to offer a service of environmental certification that specifically results in the sustainable use of natural resources, and contributes to the enjoyment of a balanced and adequate environment for the development of life. From the review made to Chanchitpricha and Bond (2013), Bond et al. (2015) and Pope et al. (2018), among
\end{abstract}


others, we distinguish six (06) dimensions that allow evaluating the effectiveness of the EIA, referring to the sustainable use of natural resources, which are: i) normative, reaching the goals established in the policies; ii) participatory, safely and reliably involving stakeholders; iii) organizational, facilitating the management of knowledge about EIA as a tool for environmental management; iv) transactional, efficiently managing resources in the execution of the EIA, such as time, finances, scope or requirements and personnel skills; v) procedural, due to a consistent and reliable procedural framework; and, vi) substantive, due to the effectiveness of the decision made by the competent authority regarding the environmental viability of the project submitted to the EIA. This paper is a contribution to further studies on the effectiveness of EIA in addressing the environmental and social problems that we currently face, and the possible trends to appear in future decades.

Keywords: Environmental Impact Assessment, Sustainability, Effectiveness, Management by Results, Public Management, Peru.

\section{Diego Espinoza Ortiz}

Magister en Gestión Pública por el Centro de Altos Estudios Nacionales, Escuela de Posgrado. Especialista en Asuntos Ambientales, Evaluación del Impacto Ambiental, Gestión y Certificación Ambiental.

Correo: Correo: despinozao@unmsm.edu.pe

\section{Gaspar Jiménez Peña}

Doctor en Desarrollo y Seguridad Estratégica por el Centro de Altos Estudios Nacionales, Escuela de Posgrado. Investigador en seguridad y defensa nacional.

Correo: inggasparajp@gmail.com

\section{José Páez Warton}

Doctor en Sociología por la Universidad Nacional Mayor de San Marcos. Investigador en ciencias sociales, seguridad y defensa nacional.

Correo: josepaez38@yahoo.es 


\section{Diego Evangelista Vargas}

Magíster en Ciencias en el Programa de Sistemática, Taxonomía Animal y Biodiversidad por la Universidad de Sao Paulo. Investigador en taxonomía, biogeografía, diversidad y conservación de la biodiversidad.

Correo: oevangelistav@unmsm.edu.pe

\section{Milward Salas Delgado}

Magíster en Derecho Constitucional y Derechos Humanos por la Universidad Nacional Mayor de San Marcos. Especialista en evaluación del impacto ambiental, gestión, certificación y fiscalización ambiental.

Correo: msalasd@unmsm.edu.pe

\section{Henrry Carrera Saavedra}

Maestría en Ciencias Ambientales de la Universidad Nacional Mayor de San Marcos. Ingeniero Ambiental y de Recursos Naturales por la Universidad Nacional del Callao. Especialista en gestión ambiental y de seguridad.

Correo: henrycs25@hotmail.com

\section{María Ángeles Natividad}

Maestría en Ciencias Ambientales de la Universidad Nacional Mayor de San Marcos. Médico Veterinario por la Universidad Alas Peruanas. Especialista en gestión ambiental y manejo de fauna.

Correo: maria.angeles.vet@gmail.com 
DIMENSIONES CONCEPTUALES PARA EVALUAR LA EFECTIVIDAD DE LA EVALUACIÓN DEL IMPACTO AMBIENTAL, BAJO LA LEGISLACIÓN AMBIENTAL PERUANA

\section{Introducción}

La Evaluación del Impacto Ambiental (en adelante, EIA) se ha convertido en una de las herramientas de gestión ambiental más notables. Fue introducida por primera vez en la Ley de Política Ambiental Nacional de los Estados Unidos en 1969, y desde entonces es ampliamente utilizada; sin embargo, también es criticada porque según algunos no estaría cumpliendo con todos sus objetivos fundamentales (Banhalmi-Zakar et al. 2018).

La EIA es un procedimiento jurídico-administrativo que tiene por objetivo la identificación, predicción e interpretación de los impactos ambientales que un proyecto o actividad produciría en caso de ser ejecutado, así como la prevención corrección y valoración de estos, todo ello con el fin de ser aceptado, modificado o rechazado por parte de las autoridades competentes (Conesa 2010).

La EIA fue implantada en la legislación peruana a través del entonces Código del Medio Ambiente y los Recursos Naturales, aprobado por decreto legislativo 613 en 1990, bajo la conducción exclusiva de los Ministerios de los sectores correspondientes a las actividades que desarrollan principalmente las empresas, según la Ley Marco para el Crecimiento de la Inversión Privada, aprobada por decreto legislativo 757 en 1991. Posteriormente, a través de la ley 27446 en 2001, se creó el Sistema Nacional de Evaluación del Impacto Ambiental (SEIA), como un sistema único y coordinado de identificación, prevención, supervisión, control y corrección anticipada de los impactos ambientales negativos derivados de las acciones humanas, expresadas por medio del proyecto de inversión, buscando el establecimiento de un proceso uniforme de evaluación y de mecanismos de participación ciudadana en el proceso.

El Reglamento de la Ley del Sistema Nacional de Evaluación del Impacto Ambiental (SEIA), aprobado por decreto supremo 019-2009-MINAM, indica que el proceso de evaluación del impacto ambiental comprende las siguientes acciones: i) clasificación del documento ambiental; ii) evaluación del documento ambiental; iii) certificación ambiental, a través de la cual se aprueba el instrumento de gestión ambiental (DIA, EIA-sd o EIA-d), certificando que el proyecto propuesto ha cumplido con los requisitos de forma 
y fondo establecidos en el marco del SEIA, con la participación del público del área de influencia y otras partes interesadas. Asimismo, se establecen las obligaciones que debe cumplir el titular para prevenir, mitigar, corregir, compensar y manejar los impactos ambientales negativos a generarse.

Hasta la fecha, vino dándose una evolución regulatoria, incorporando progresivamente acciones y requisitos provenientes de buenas prácticas ambientales internacionales y múltiples lecciones aprendidas, que reflejan una cultura social reactiva y con limitado enfoque estratégico.

En ese sentido, es oportuno revisar la efectividad de la EIA, para abordar los problemas ambientales y sociales actuales, así como las tendencias en las futuras décadas. Para ello se precisa recopilar y analizar información de los procedimientos de la evaluación de impacto ambiental para construir un estado del arte de la EIA, debido a las limitadas estadísticas generales y recopilaciones de datos oficiales (Cannaos \& Onni 2019). Sin embargo, de acuerdo con Banhalmi-Zakar et al. (2018), encontramos dos alternativas para transcurrir por este sendero: i) continuar la «evolución» de la EIA, manteniendo intactos la mayoría de sus elementos, actividades y subprocesos, pero con nuevas formas de superar los desafíos y limitaciones; o ii) enrumbarse en la «revolución» de la EIA, buscando que todo el proceso actual sea reemplazado. Por tanto, el objetivo es saber cuáles deben ser los indicadores a emplear para determinar la efectividad de la EIA en el Perú, a efectos de potenciar esta herramienta de gestión hacia el uso sostenible de los recursos naturales en el contexto actual y venidero.

\section{Marco teórico}

La efectividad de la EIA es la capacidad de lograr el efecto que se desea o se espera de la misma. Eso se alcanza logrando el uso sostenible de los recursos naturales para el goce de un ambiente equilibrado y adecuado al desarrollo de la vida, y a consecuencia de esto la ciudadanía satisface su interés general por saber cuáles son los impactos ambientales significativos de las actividades realizadas en el territorio nacional.

Respecto a la efectividad de la EIA, Sadler (1996) la define como la medida en que este proceso contribuye a sus objetivos a largo plazo, es decir, la protección del medio ambiente o, aún más ambicioso, el desarrollo sostenible. A mayor detalle, Bond, Pope y Morrison-Saunders (2015) indican que la efectividad de la EIA tiene seis dimensiones: procedimental, sustantiva, transaccional, normativa, de pluralismo, y de conocimiento y aprendizaje. Según lo reportado por Sadler (1996) y Baker y McLelland (2003), la efectividad procedimental considera los principios y la práctica del proceso de 
evaluación de impacto y puede medirse con base en la práctica de evaluación de impacto que muestra cómo se aplica la política en el proceso; asimismo, la efectividad sustantiva puede medirse en función del desempeño en relación con el logro de los objetivos de la evaluación de impacto en la práctica; y la efectividad transactiva se logra cuando los recursos, en términos de recursos humanos, costo y tiempo, se invierten al nivel mínimo para lograr los objetivos establecidos o resultados eficientes.

Por su parte, Bond, Morrison-Saunders y Howitt (2013) señalan que la efectividad normativa está relacionada con el sentido de los principios que la sociedad (o una comunidad) acuerda a través de la normativa y las reglas aceptadas dentro símisma, por ejemplo, la promoción del desarrollo sostenible; mientras que la efectividad relacionada con el conocimiento y aprendizaje en la EIA plantea la posibilidad de fortalecer el conocimiento sobre el desarrollo sostenible en las partes interesadas para lograr el mismo; y el pluralismo reconoce la variedad de opiniones del público respecto a la evaluación del impacto y cómo estas aportan al mismo. Es así como, considerando el objetivo y el marco teórico de la presente investigación, se considera la pertinencia de determinar los indicadores de cada dimensión de la efectividad de la EIA.

\section{Método}

Se utilizó el método de estudio de caso para desarrollar esta investigación cualitativa, centrada en la EIA como herramienta de gestión, en el intento de conocer cómo funcionan todas las partes que la componen y las relaciones entre ellas para formar un todo (Ramos 2007). Para ello, se utilizó básicamente la técnica de análisis documental, abarcando la normativa nacional e internacional, así como publicaciones especializadas, sobre la evaluación del impacto ambiental. Esta información fue analizada de forma sistemática, con la finalidad de registrar, objetivamente, la situación del conocimiento de las características para determinar la efectividad de la EIA.

\section{Resultados}

\subsection{Efectividad de la EIA y su cadena de valor}

En el Perú existen normas que respaldan la implementación de la gestión por procesos orientadas a resultados, con el fin de satisfacer las necesidades de las partes interesadas, como la Política Nacional de Modernización de la Gestión Pública, aprobada por decreto supremo 004-2013-PCM. De acuerdo con 
ello, la efectividad de la EIA debe entenderse sobre la base de una cadena de valor para la gestión por resultados (Figura 1), con los siguientes elementos:

a) Insumo: El recurso que se usa para la producción y entrega de los productos. Destrezas y competencias que apuntalan el proceso de la EIA, incluye tiempo y costos para la implementación de la EIA.

b) Proceso: Conjunto de actividades coordinadas y controladas llevadas a cabo para lograr un producto y/o servicio deseado, conforme con requisitos específicos, incluyendo las limitaciones del estudio, tiempo, costo y recursos de los EIA. Proceso de cómo la EIA está en la regulación.

c) Producto: Es lo que se entrega al público o sociedad. Son los documentos de Certificación Ambiental que contienen información para la toma de decisiones informadas sobre las acciones propuestas con impactos ambientales.

d) Resultado específico: Lo que se desea lograr. Consecuencia de lograr un producto específico. Decisiones informadas con las obligaciones derivadas de la certificación ambiental para las actividades futuras (autorizaciones). Dar efecto a los principios de la legislación ambiental.

e) Resultado final: Es lo que se pretende obtener o cambiar en la realidad. Impacto al desarrollo tras lograr resultados específicos. Realización progresiva del derecho reconocido en la constitución.

Figura 1. Cadena de valor de la EIA

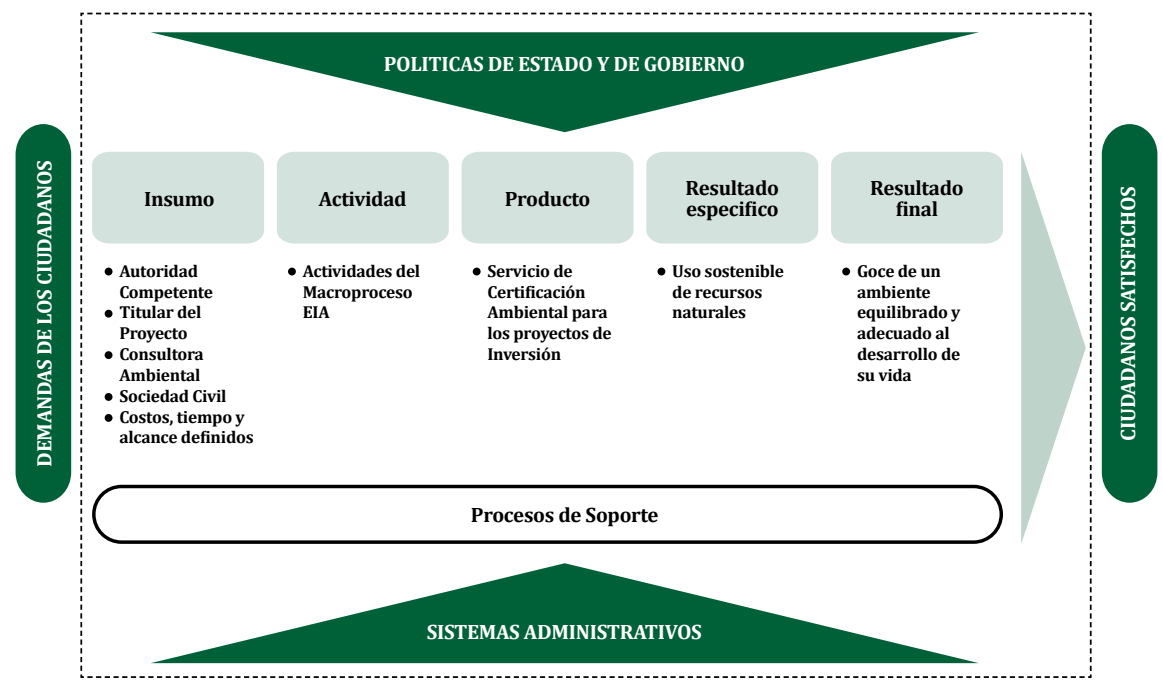


DIMENSIONES CONCEPTUALES PARA EVALUAR LA EFECTIVIDAD DE LA EVALUACIÓN DEL IMPACTO AMBIENTAL, BAJO LA LEGISLACIÓN AMBIENTAL PERUANA

\subsection{Dimensiones de la efectividad de la EIA}

De la revisión efectuada para la presente investigación cualitativa, en especial de las dimensiones definidas por Chanchitpricha y Bond (2013), Bond, Pope y Morrison-Saunders (2015), y Pope, Bond, Cameron, Retief y MorrisonSaunders (2018), se proponen las siguientes dimensiones para la evaluación de la efectividad de la EIA en el Perú: normativa, participativa, organizacional, transaccional, procedimental y sustantiva.

\section{a) Efectividad normativa}

Capacidad de lograr el uso sostenible de los recursos naturales, alcanzando las metas políticas, normativas y estratégicas, que son: invertir las tendencias prevalecientes (insostenibles), integrar todos los factores clave entrelazados que afectan a la sostenibilidad, buscar beneficios que se refuercen mutuamente, minimizar las compensaciones, respetar los contextos en los que se lleva a cabo la evaluación de la sostenibilidad, entre otros.

La efectividad normativa resulta de la legitimidad de la autoridad competente al conducir el macroproceso y las actividades de la EIA, y ofrecer el servicio de certificación ambiental, acorde al desarrollo o cambios en las políticas institucionales, normas, evaluaciones ambientales estratégicas y demás instrumentos de gestión.

\section{b) Efectividad participativa}

Capacidad de lograr el uso sostenible de los recursos naturales, involucrando de forma segura y confiable a las partes interesadas, resultado de la equidad y la aceptabilidad del proceso de participación ciudadana en el servicio de certificación ambiental.

La equidad es la condición básica para la confianza en la participación ciudadana, para un servicio transparente y accesible, recogiendo aprendizajes, percepciones y lecciones aprendidas a través de los diversos mecanismos y canales de atención.

La aceptabilidad es la seguridad de considerar los aportes u opiniones del público en la participación ciudadana, para influir en la toma de decisión durante el servicio de certificación, gracias al intercambio amplio y oportuno de información en la elaboración y evaluación del estudio ambiental.

\section{c) Efectividad organizacional}

Capacidad de facilitar la gestión del conocimiento sobre la EIA como instrumento para el uso sostenible de recursos naturales, resultado del liderazgo transformacional y del mantenimiento de capacidad técnica de la autoridad 
competente para conducir el macroproceso y las actividades de la EIA y ofrecer el servicio de certificación ambiental.

El liderazgo transformacional es la capacidad de adaptar prácticas individuales, profesionales y organizativas, reorganizando equipos de trabajo, como parte de un proceso de aprendizajes y lecciones aprendidas sobre la EIA, con miras a ofrecer un servicio con equipos orientados al beneficio del usuario. El mantenimiento de capacidad técnica está referida a los profesionales de la autoridad competente y su disposición para reconocer y comprender las necesidades de los usuarios, actuando con la pericia y profesionalismo a medida de cada caso, y demostrar integridad y solvencia técnica en la ejecución del servicio.

\section{d) Efectividad transaccional}

Capacidad de lograr el uso sostenible de los recursos naturales, resultado de la gestión eficiente de recursos para ejecutar la EIA y ofrecer el servicio de certificación ambiental, acorde al desarrollo o cambios en las políticas institucionales y demás pertinentes.

Desde la perspectiva de la administración pública, los recursos a considerar son el tiempo, los recursos financieros, el alcance y la habilidad del personal (tabla 1).

Tabla 1. Indicadores de la efectividad transaccional de la cadena de valor de la EIA

\begin{tabular}{|cccc}
\hline Insumo & Actividad de la EIA & $\begin{array}{c}\text { Servicio de } \\
\text { certificación ambiental }\end{array}$ & $\begin{array}{c}\text { Uso sostenible de } \\
\text { recursos naturales }\end{array}$ \\
\hline Tiempo & $\begin{array}{c}\text { Emisión de documentos } \\
\text { con celeridad }\end{array}$ & $\begin{array}{c}\text { Con cumplimiento de } \\
\text { plazos }\end{array}$ & Oportuno \\
\hline Recurso financiero & $\begin{array}{c}\text { Evaluación de fondo } \\
\text { a los documentos } \\
\text { presentados }\end{array}$ & Con gasto razonable & Económico \\
\hline Alcances definidos & $\begin{array}{c}\text { Coordinación para } \\
\text { cumplir actividades, } \\
\text { funciones y requisitos }\end{array}$ & $\begin{array}{c}\text { Con cumplimiento del } \\
\text { alcance definido }\end{array}$ & Cumpliendo alcance \\
definido
\end{tabular}

Fuente: Elaboración propia.

\section{e) Efectividad procedimental}

Capacidad de lograr el uso sostenible de los recursos naturales, debido a un marco procedimental coherente y fiable para ofrecer el servicio de certificación ambiental, basado en información con calidad, conformidad y excelencia. 
La calidad se relaciona con un marco procedimental uniforme que permita a la autoridad competente acompañar la elaboración del estudio ambiental, para que este se base en información fidedigna, comprensible y acorde a los sistemas de información ambiental (geoservidores, redes de monitoreo y vigilancia ambiental, instrumentos de planificación, entre otros); y después ofrecer un servicio de certificación ambiental que evalúe a fondo las predicciones y argumentos del estudio elaborado, con rigurosidad y proporcionalidad, sobre los puntos críticos para la toma de decisión.

La conformidad se relaciona con un marco procedimental uniforme que permita al titular del proyecto y a la consultora ambiental evaluar preliminarmente los impactos ambientales de la acción propuesta (scoping), personalizando la información técnica básica para elaborar el estudio ambiental, y cumplir el contenido mínimo de los términos de referencia en la etapa de admisibilidad del servicio de certificación ambiental.

La excelencia se relaciona con un marco procedimental uniforme que permita al titular del proyecto y a la consultora ambiental incorporar las mejores prácticas ambientales en el estudio ambiental, a fin de contribuir con la mejora continua del servicio de certificación ambiental.

\section{f) Efectividad sustantiva}

Capacidad de lograr el uso sostenible de los recursos naturales, debido a la eficacia de la decisión tomada por la autoridad competente respecto de la viabilidad ambiental del proyecto, con el intercambio continuo de información y la intervención de entidades especializadas en el servicio de certificación ambiental.

La toma de decisiones informadas implica utilizar los documentos producidos como parte del macroproceso y de las actividades de la EIA; y considerar sustancialmente la intervención de entidades especializadas y el diálogo continuo entre las partes involucradas. 
Revista KaWSAypacha. Sociedad y MedioAmbiente Nº 8 (2021)

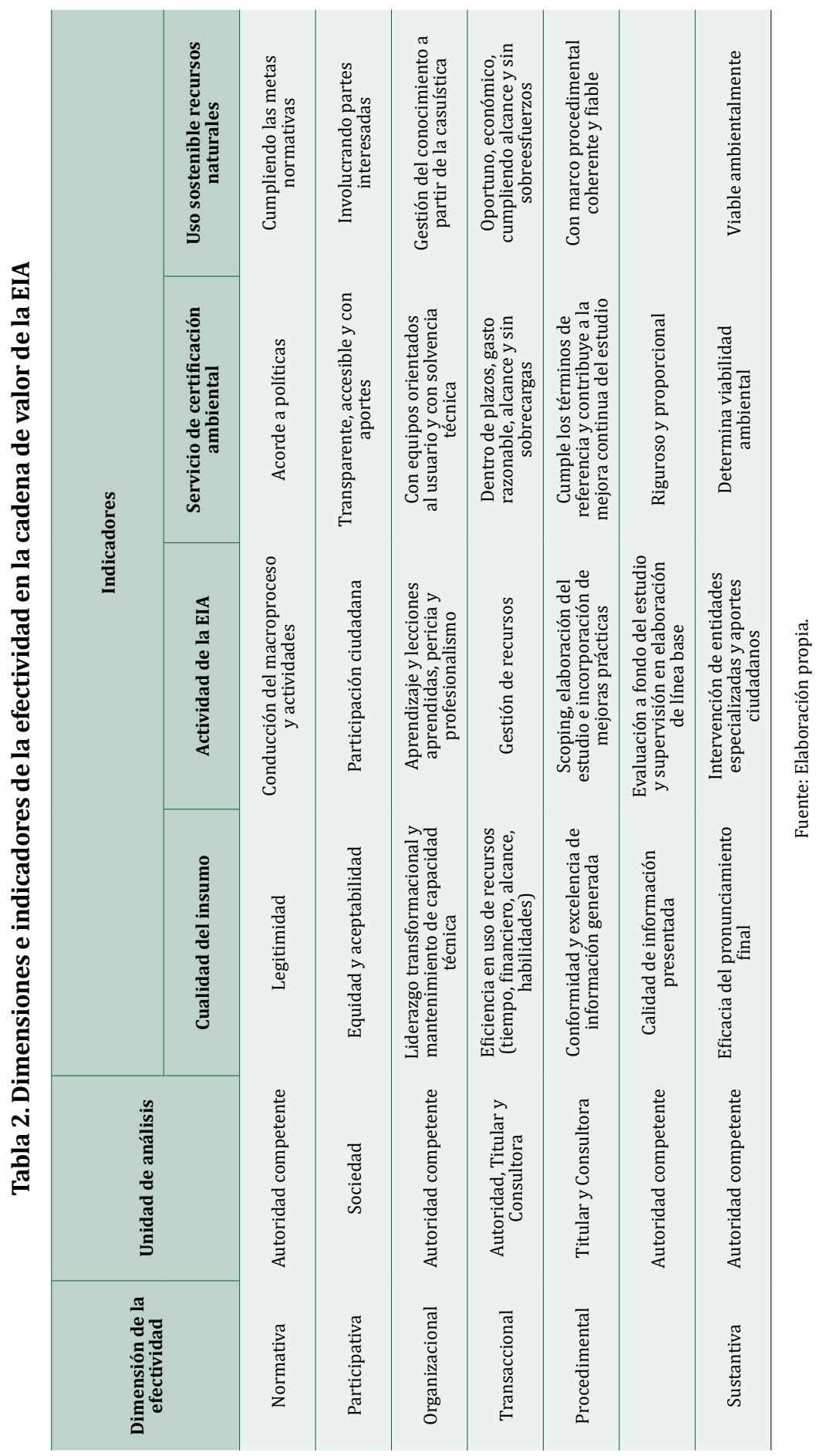


DIMENSIONES CONCEPTUALES PARA EVALUAR LA EFECTIVIDAD DE LA EVALUACIÓN DEL IMPACTO AMBIENTAL, BAJO LA LEGISLACIÓN AMBIENTAL PERUANA

\section{Discusión}

La efectividad de la EIA es la capacidad de esta herramienta para lograr el uso sostenible de los recursos naturales, contribuyendo a un ambiente equilibrado y adecuado para la vida. Esto coincide con Sadler (1996), quién definió la efectividad como qué tan bien funciona algo o si funciona según lo previsto y cumple con los propósitos para los que fue diseñado. En ausencia de un marco conceptual consistente y objetivo de los indicadores para la determinación de la efectividad de la EIA en el Perú, esta investigación cualitativa efectuó un análisis documental de fuentes oficiales y de investigaciones para enfocar el problema sobre la base de una cadena de valor para la gestión por resultados en la administración pública, toda vez que el macroproceso de la EIA es conducido por el Estado a través de las autoridades ambientales competentes.

La cadena de valor desarrollada en la presente investigación (Figura 1) sirve para distinguir conceptualmente, desde el punto de vista de la gestión pública, cuáles son los insumos y las actividades en el macroproceso de la EIA, cuyo producto es el servicio de certificación ambiental ofrecido; y distinguirlos respecto del resultado específico y del resultado final en la realidad. Este modelo de cadena de valor general para la EIA guarda relación con el marco conceptual desarrollado por Alberts, Retief, Roos, Cilliers y Arakele (2019), con indicadores para la evaluación de un sistema de EIA.

La investigación distingue seis dimensiones que constituyen la cadena de valor para la efectividad de la EIA en el Perú: normativa, participativa, aprendizaje organizacional, transaccional, procedimental y sustantiva. Estas dimensiones fueron determinadas en la presente investigación, en concordancia con aquellas definidas por Bond, Pope y Morrison-Saunders (2015), que indican que la efectividad tiene seis dimensiones: procedimental, sustantiva, transaccional, normativa, de pluralismo, de conocimiento y aprendizaje, las cuales guardan relación con los trabajos de Chanchitpricha y Bond (2013), Shakil y Ananya (2015), Kolhoff et al. (2016), Günther, Geibler y Köppel (2017), Bond et al. (2018), Pope et al. (2018), Zhang, Kørnøv y Christensen (2020) y Khan, Chaudhry, Ahmad, Mehmood y Saif (2020), que recogen objetivamente la experiencia en la performance, la calidad y la efectividad de la EIA en diversas partes del mundo. Asimismo, las dimensiones responden a las recomendaciones de Nita (2019) para potenciar el conocimiento de las evaluaciones de impacto ambiental, enfocar sus principales beneficios y problemas, y mejorar la calidad y eficacia de los procesos formales; de la Comisión para el Desarrollo Minero Sostenible (2020), en los ejes de entorno social: ciudadanía, diversidad y territorio, gestión ambiental y mejora regulatoria; y de Espinoza (2020), en la calidad de certificación ambiental para los proyectos energéticos para abordar los 
problemas ambientales y sociales que ahora enfrentamos y las tendencias en las futuras décadas.

\section{a) Efectividad normativa}

La efectividad normativa es la capacidad de lograr el uso sostenible de los recursos naturales acorde al desarrollo o cambios en las políticas institucionales y normativa pertinente, resulta de la legitimidad de la autoridad competente al conducir el macroproceso de la EIA y ofrecer el servicio de certificación ambiental. Esto concuerda con Bond y Morrison-Saunders (2013), quienes consideran que la efectividad normativa abarca las percepciones o las actitudes de la sociedad respecto a la EIA, cumpliendo las normas sociales e institucionales que plasman las perspectivas o los patrones de conocimiento entorno a los procesos de evaluación de impacto y su contribución para mejorar la calidad de vida.

Entre las políticas, a través del decreto supremo 023-2021-MINAM, se aprobó la Política Nacional del Ambiente al 2030 como principal instrumento orientador y de más alto nivel que define y conduce las acciones de las entidades del Estado para av anzar hacia un ambiente sano y seguro para todas y todos los peruanos. En ella, se desarrollan los ejes necesarios para afrontar los principales problemas ambientales del país de forma articulada desde todos los niveles de gobierno, aspirando al bienestar de la población, a través de la mejora en la provisión de bienes y servicios ecosistémicos para el desarrollo de las personas y la sostenibilidad ambiental. A efectos de cumplir estos fines, la política propone entre sus objetivos fortalecer la gobernanza ambiental en los tres niveles de gobierno, la gestión del conocimiento ambiental de entidades públicas y privadas, y el uso sostenible de las especies de flora y fauna.

En relación con las metas normativas necesarias, la Comisión para el Desarrollo Minero Sostenible (2020) recomienda que para la gestión ambiental deberían tenerse estándares idóneos para proteger nuestro entorno y dar predictibilidad a las decisiones del sector privado, así como una política clara de compensación ambiental e instrumentos que permitan su debida aplicación. Además, se recomienda que, para el cumplimiento de obligaciones sociales, se aprueben protocolos y guías referenciales que permitan asegurar la calidad de estos compromisos y facilitar su debida fiscalización; incluso, la Defensoría del Pueblo (2020) recomienda actualizar los dispositivos normativos relacionados con la protección del derecho a la participación ciudadana.

La Asociación Internacional para la Evaluación del Impacto (IAIA 2020) señala que a medida que nos adentramos en el siglo XXI, se reconoce cada vez más que la transición a una economía mundial con bajas emisiones 
de carbono para 2050 requerirá un desarrollo de infraestructura masivo y rápido, mucho más allá de lo que experimentó el mundo en épocas anteriores. Asimismo, se señala que los encargados de formular políticas y tomar decisiones deberían confiar más en los profesionales de la evaluación de impacto ambiental para aportar una visión de sostenibilidad e inclusión a estos esfuerzos, bajo los siguientes objetivos: i) gestionar el consumo de las poblaciones y reducir el consumo de energía y recursos mediante la eficiencia y la adopción de un modelo económico regenerativo y circular; ii) reducir la pobreza a través de sistemas económicos más equitativos que tengan en cuenta la asistencia a las naciones en desarrollo, los pueblos indígenas y las poblaciones subrepresentadas; iii) restaurar el hábitat a través del aumento de la biodiversidad y la resiliencia, así como la valoración de los servicios de los ecosistemas; iv) abordar el cambio climático adoptando compromisos estrictos de descarbonización -que reducen las emisiones de gases de efecto invernadero- y acelerar el despliegue de tecnologías de energía renovable; y v) eliminar los químicos tóxicos persistentes que ingresan al medio ambiente en forma de metales pesados, plásticos y compuestos orgánicos complejos que actúan como disruptores endocrinos; entre otros.

Para la realidad peruana, estas condiciones de contexto pueden abordarse de forma más efectiva a través de la aplicación de la Evaluación Ambiental Estratégica (EAE) con un auténtico enfoque estratégico, definida por el Ministerio del Ambiente (2021) como instrumento de naturaleza estratégica que ayuda a crear un contexto de desarrollo para la sostenibilidad mediante la integración de aspectos ambientales y de sostenibilidad, agregando valor en la toma de decisiones, al evaluar las opciones de desarrollo estratégico en relación con las condiciones de contexto. Esto refuerza la mirada de la EIA como un instrumento de gestión dirigido al control efectivo de los impactos de un proyecto, dejando a la EAE como un instrumento estratégico dirigido a la construcción de futuros a través de las políticas, planes y programas.

\section{b) Efectividad participativa}

La efectividad participativa es la capacidad de lograr el uso sostenible de los recursos naturales, involucrando de forma segura y confiable a las partes interesadas, resultado de la equidad y la aceptabilidad de la participación ciudadana en el servicio de certificación ambiental, a través de los mecanismos de participación ciudadana. La noción de pluralismo conduce al estudio de las opiniones y los aportes ciudadanos, a través de los cuales diferentes partes interesadas comparten sus puntos de vista respecto a un proyecto y sus impactos ambientales (Pope, Annandale y Morrison-Saunders 2004). En relación con esta dimensión, la Comisión para el Desarrollo Minero Sostenible (2020) recomienda, respecto a la participación ciudadana, la necesidad de 
incluir mayores mecanismos participativos que involucren un diálogo abierto con la ciudadanía desde las etapas tempranas de identificación, diseño y desarrollo de los proyectos; y fortalecer los mecanismos de intervención temprana, así como los de transparencia y acceso a la información, de las autoridades ambientales.

Esto es también acorde a los resultados de Espinoza (2020), que recomienda además considerar indicadores adicionales de los enfoques de interculturalidad, de género e intergeneracional en próximas investigaciones sobre la efectividad de la participación ciudadana, a fin de integrar el pluralismo en la toma de decisiones, considerando la opinión de los grupos de interés como unidades de información. Además, la Defensoría del Pueblo (2020) recomienda la modificación de dispositivos normativos sectoriales, destinada a la protección del derecho a la participación ciudadana, a fin de que contemplen como mecanismos de participación ciudadana en la gestión ambiental únicamente aquellos que efectivamente permitan a la ciudadanía formular sus aportes y observaciones para la mejora en la toma de decisiones, garantizando en todos los casos un adecuado y eficaz ejercicio de este derecho, sin perjuicio de garantizar el derecho de acceder a la información ambiental.

La participación ciudadana, a la fecha, debe considerar tanto los escenarios presenciales con la opinión de la autoridad sanitaria, como los más oportunos mecanismos no presenciales que reducen el riesgo de contagio del COVID-19, en aplicación del decreto legislativo 1500, por medios electrónicos, virtuales u otro medio de comunicación, con las siguientes condiciones básicas: acceso a energía eléctrica, adecuado nivel de acceso, acceso a los equipos de telecomunicación, habilidades de la población participante para utilizar los equipos y tecnologías electrónicas (virtuales u otros), así como la previsión de medidas destinadas a asegurar dichas habilidades, y complementariedad entre los medios aprobados para la implementación de los mecanismos de participación ciudadana.

Una vez superados los retos para la implementación de mecanismos para una participación ciudadana efectiva, perdura la necesidad de establecer mecanismos de monitoreo y evaluación de la ejecución de estos, a fin de asegurar que los ciudadanos interesados gocen de una participación ciudadana efectiva. Relacionado con ello, la Comisión para el Desarrollo Sostenible (2020) anota recomendaciones respecto a los acuerdos sociales que pudieran derivarse, como fortalecer las capacidades de las comunidades para lograr la adopción de acuerdos que contribuyan eficazmente a su desarrollo, y contar con mecanismos de monitoreo, supervisión y resolución de conflictos. Aun así, existen situaciones críticas donde es necesario incluir estrategias más especializadas de participación, sobre todo cuando 
existe el potencial de reasentamiento de poblaciones, donde es necesario contar con reglas claras y vinculantes para el sector minero que asegure el bienestar y respeto de los derechos de la población desplazada, y considerar explícitamente un conjunto de estándares internacionales.

\section{c) Efectividad organizacional}

La efectividad organizacional es la capacidad de facilitar la gestión del conocimiento sobre la EIA como instrumento para el uso sostenible de recursos naturales, resultado del liderazgo transformacional y del mantenimiento de capacidad técnica de la autoridad competente para conducir el macroproceso de la EIA y ofrecer el servicio de certificación ambiental. De acuerdo con varios investigadores, el conocimiento y aprendizaje respecto de la EIA sí contribuye a resultados efectivos de esta herramienta (Nilsson 2005, Jha-Thakur, Gazzola, Peel, Fischer \& Kidd 2009), siendo el aprendizaje lo que conduce a un cambio en el diseño de políticas o proyectos para ofrecer mejores resultados sostenibles, y el conocimiento refiere al cambio en las creencias con consecuencias más amplias para la forma en que los profesionales y público interesado se comportan en torno al macroproceso de la EIA.

La Comisión para el Desarrollo Minero Sostenible (2020) recomienda fortalecer las capacidades humanas, de gestión y financieras de las entidades involucradas en el proceso de evaluación ambiental, incluso regulando un Plan Integral de Fortalecimiento Institucional o un Plan de Modernización en coordinación con todas las entidades públicas, de los tres niveles de gobierno, que intervengan en cualquier procedimiento administrativo, de cualquier naturaleza, a lo largo de la cadena de valor de la actividad minera.

Asimismo, Espinoza (2020) también recomienda que es necesario mantener la mejora continua de las plataformas informáticas, articulando los registros georreferenciados de certificaciones ambientales, guías e instrumentos de planificación; es más, puede ser pertinente aspirar a que la labor administrativa y técnica se realice dentro de una interfaz virtual sin que haya necesidad de descargar archivos a un ordenador.

Sobre este punto, el Instituto de Gestión y Evaluación Ambiental del Reino Unido (IEMA 2020) recomienda la implementación de la evaluación de impacto ambiental completamente en medio digital, a través de una solución de interfaz o software de interoperabilidad bajo el estándar ISO 19650 sobre modelado de información de construcción o BIM (Building Information Modeling), con un entorno de datos comunes (common data) o entorno colaborativo, para una mejor gestión de la evaluación de impacto en soporte digital. Es importante que los resultados de esta evaluación del impacto deban ser comunicados de manera efectiva, con herramientas de visualización y 
generación de reportes automatizados, sistemas web a la medida, tableros de control, sistemas de información geográfica, uso de modelos de realidad virtual o realidad aumentada, entre otros.

\section{d) Efectividad transaccional}

La efectividad transaccional es la capacidad de lograr el uso sostenible de los recursos naturales debido a la gestión eficiente de recursos para ejecutar la EIA y ofrecer el servicio de certificación ambiental, acorde al desarrollo o cambios en las políticas institucionales y demás pertinentes. De forma tradicional, se considera que la efectividad transaccional está en función a la inversión de tiempo y dinero como factores clave; sin embargo, Theophilou, Bond y Cashmore (2010) consideran, adicionalmente, a las categorías de especificación de roles (alcance) y habilidades, que, junto al tiempo y a los recursos financieros, reflejan cómo se invierten los recursos en la EIA.

De acuerdo con Espinoza (2020), la efectividad transaccional debe reconocer: i) el costo-beneficio a la nación a través de la valorización económica de los impactos, básicamente por daños evitados al ambiente y beneficios en los servicios ambientales, adicionales siempre a la pérdida neta cero, que contribuyan al desarrollo local; y ii) su relación con la cantidad de recursos utilizados en la conducción del servicio de certificación ambiental.

Considerando que la concepción del ecosistema guarda mayor relación con el sistema complejo adaptativo, es necesario que los insumos necesarios para la evaluación del impacto ambiental tengan similar perspectiva en su gestión, por lo que no es recomendable trabajar bajo un alcance restringido, sino más bien en un modelo de gestión ágil, estableciendo restricciones en gasto y plazos de los procedimientos y manejo adaptativo en el cumplimiento del alcance. De acuerdo con Sánchez y Falero (2015), se ha evidenciado una contradicción entre una concepción del ecosistema, que está muy cerca de lo que es un sistema complejo adaptativo, y los instrumentos que se usan para su gestión, que no reconocen esa condición, lo cual tendría su base en la escasa reflexión epistemológica respecto de la elaboración de la normativa ambiental, como también en un paradigma que no reconoce la dinámica constante dentro de un ecosistema.

En esa misma línea, resulta necesario que el esfuerzo del personal y la inversión de tiempo migren progresivamente de un enfoque de obrero a un enfoque de gestor, donde es más importante tomar decisiones intermedias dentro la especialidad que estar invirtiendo recursos en, mayoritariamente, leer documentos. Es necesario desarrollar esquemas operativos que apoyen los procesos de evaluación de impacto ambiental, lo cual coincide con el modelo de evaluación de impacto digital propuesto por el Instituto de Gestión y Evaluación Ambiental del Reino Unido (IEMA 2020), que sugiere el desarrollo 
de habilidades de gestión digital, como gestión, procesos y seguimiento de datos, herramientas de automatización, modelado de información de construcción (BIM, por sus siglas en inglés), entorno de datos común, registro de datos, bases de datos, entre otros.

La Comisión para el Desarrollo Minero Sostenible (2020) coincide con esto, al recomendar que para la fiscalización ambiental se incorporen de manera progresiva herramientas tecnológicas para la fiscalización ambiental, lo cual llevaría a repensar el enfoque de la fiscalización y diseñar nuevos mecanismos que aseguren el cumplimiento de las obligaciones ambientales. Incluso, la Comisión llega a recomendar que como mejora regulatoria se establezca una ventanilla única multisectorial de los trámites necesarios para el desarrollo de un proyecto. Un reciente avance en esta línea es el Plan BIM como parte del Plan Nacional de Competitividad y Productividad, aprobado el 28 de julio de 2019, mediante el decreto supremo 237-2019-EF, que busca garantizar una adecuada ejecución de las inversiones de edificaciones e infraestructura desde el sector público, incorporando procesos, metodologías y tecnologías de información a lo largo del ciclo de inversión. Asimismo, el Plan BIM Perú busca fomentar un articulado proceso de adopción por parte del sector público, acompañado del sector privado y la academia.

\section{e) Efectividad procedimental}

La efectividad procedimental es la capacidad de lograr el uso sostenible de los recursos naturales, debido a un marco procedimental coherente y fiable, para ofrecer el servicio de certificación ambiental, basado en información con calidad, conformidad y excelencia. Según la bibliografía, los factores que influyen en la eficacia de los procedimientos podrían ser el marco de políticas, el contexto político, la disponibilidad de recursos, la participación pública activa, y el conocimiento y la experiencia que poseen los profesionales de la evaluación del impacto ambiental. Esta dimensión está relacionada con la calidad de la información utilizada en el proceso único de evaluación del impacto ambiental, que debe ser suficientemente comprensivo para fortalecer la revisión en los puntos críticos del impacto ambiental de un determinado proyecto.

En relación con esta dimensión, Espinoza (2020) recomienda que se impulse el análisis de alternativas del proyecto en la etapa de evaluación ambiental preliminar y aprobación de términos de referencia específicos, a efectos de brindar mayor predictibilidad en el contenido mínimo necesario para admitir los estudios ambientales con mayor dedicación sobre los aspectos significativos del proyecto, el diseño del proyecto a nivel de ingeniería básica, las fases tempranas de la jerarquía de mitigación (prevención y mitigación por el diseño de proyecto), datos e información de campo obtenidos en la línea base. Esto conllevaría a que la revisión de fondo efectuada por la autoridad 
competente sea más rigurosa en la caracterización del impacto y la estrategia de manejo ambiental, permitiendo establecer indicadores de seguimiento y control (follow-up) más realistas y cercanos al cumplimiento.

Respecto al volumen de información clave para evaluar los impactos, la Comisión para el Desarrollo Minero Sostenible (2020) aborda la problemática de los EIA enciclopédicos, destacando la necesidad de contar con estudios que prioricen información relevante y den mayor énfasis al análisis técnico de impactos; a lo cual también es oportuna la interconexión de información generada por diferentes entidades del Estado y empresas, dentro de la cual se encuentra el Registro Administrativo de Certificaciones Ambientales, con información cierta y completa sobre las actividades. Frente a esta problemática, es pertinente señalar el modelo de gestión propuesto por el Instituto de Gestión y Evaluación Ambiental de Reino Unido, que relaciona el uso de datos para la evaluación del impacto a través de la recopilación en aplicativos móviles de información geográfica, en drones, el uso de sensores remotos, teledetección y monitoreo automatizado.

\section{f) Efectividad sustantiva}

La efectividad sustantiva es la capacidad de lograr el uso sostenible de los recursos naturales, debido a la eficacia de la decisión tomada por la autoridad competente respecto de la viabilidad ambiental del proyecto, con el intercambio continuo de información y la intervención de entidades especializadas en el servicio de certificación ambiental. Esto puede contribuir a la gestión integrada de los recursos naturales a través de la prevención de impactos acumulativos y a la supervisión de la eficacia de las medidas de manejo ambiental aprobadas. La literatura sugiere que el logro de una efectividad sustantiva podría depender de varios factores, como el marco regulatorio para implementar la evaluación de impacto en el proceso de toma de decisiones, el mecanismo en el contexto de toma de decisiones, la participación pública y la calidad en la evaluación del estudio. No obstante, es notorio que la variable ambiental viene dejando de ser vista como un costo para las empresas y como un obstáculo para las inversiones por parte de los países, siendo ahora un factor más bien para la competitividad.

En relación con esta dimensión, la Comisión para el Desarrollo Minero Sostenible (2020) indica la necesidad de una intervención temprana con el acompañamiento de la autoridad ambiental, como parte de un nuevo enfoque de la evaluación ambiental, temprana y concurrente, junto a la opinión técnica especializada de entidades públicas intervinientes, para reforzar lo sustancial de las recomendaciones técnicas sobre los aspectos claves del estudio, conforme estos se vayan definiendo. De esta manera se contribuye a que la certificación ambiental no sea vista como un «permiso» más, sino como una herramienta para la protección ambiental y la sostenibilidad. 
Se considera que deberían existir mayores estudios académicos de ese tipo, considerando la casuística sobre la efectividad en las decisiones concretas sobre la evaluación del impacto ambiental, dado que estos se relacionan directamente con actividades en cuyo ciclo de vida se generan impactos ambientales y sociales negativos significativos, para contribuir al goce de un ambiente equilibrado y adecuado para el desarrollo de la vida.

\section{Conclusión}

La efectividad de la EIA es la capacidad de esta herramienta para lograr el uso sostenible de los recursos naturales, contribuyendo a un ambiente equilibrado y adecuado para la vida. La efectividad puede representarse a través de un modelo conceptual de su cadena de valor, donde los insumos son las partes intervinientes y los recursos; las actividades corresponden al macroproceso de la EIA; el producto es el servicio de certificación ambiental ofrecido para los proyectos; el resultado inmediato es permitir el uso sostenible de los recursos naturales; y el resultado final es contribuir al goce de un ambiente equilibrado y adecuado para la vida. La efectividad de la EIA en el Perú puede evaluarse desde seis dimensiones propuestas y descritas: normativa, participativa, organizacional, transaccional, procedimental y sustantiva, tal y como lo propone el presente artículo académico. 


\section{Referencias}

Alberts, RC; Retief, FP; Roos, C; Cilliers, DP; \& Arakele, M. (2019). Re-thinking the fundamentals of EIA through the identification of key assumptions for evaluation. Impact Assessment and Project Appraisal. https://doi.org/10.1080 /14615517.2019.1676069

Baker, D. C. \& McLelland, J. N. (2003). Evaluating the effectiveness of British Columbia's environmental assessment process for first nations' participation in mining development. Environmental Impact Assessment Review, 23(5), 581-603.

Banhalmi-Zakar, Z.; Gronow, C.; Wilkinson, L.; Jenkins, B.; Pope, J.; Squires, G.; Witt, K.; Williams, G. y Womersley, J. (2018). Evolution or revolution: where next for impact assessment? Impact Assessment and Project Appraisal, 36(6), 506515. https://doi.org/10.1080/14615517.2018.1516846

Bond, A. \& Morrison-Saunders, A. (2013). Challenges in determining the effectiveness of sustainability assessment. En: A. Bond, A. Morrison-Saunders y R. Howitt (editores), Sustainability Assessment: Pluralism, Practice and Progress (pp. 3750). London: Taylor \& Francis.

Bond, A.; Morrison-Saunders, A.; Howitt, R. (2013). Framework for comparing and evaluating sustainability assessment practice. En: A. Bond, A. MorrisonSaunders y R. Howitt (editores). Sustainability Assessment: Pluralism, Practice and Progress (pp. 117-131). London: Taylor \& Francis.

Bond, A.; Pope, J.; Morrison-Saunders, A. (2015). Introducing the roots, evolution and effectiveness of sustainability assessment. En: Morrison-Saunders, A., Pope, J. and Bond, A. (editores.). Handbook of sustainability assessment (pp. 3-19). UK: Edward Elgar Publishing, Cheltenham.

Bond, A.; Retief, F.; Cave, B.; Fundingsland, M.; Duinker, P.N.; Verheem, R. y Brown, A.L. (2018). A contribution to the conceptualisation of quality in impact assessment. Environmental Impact Assessment Review, 68, 49-58. https://doi.org/10.1016/j.eiar.2017.10.006

Cannaos, C.\& Onni, G. (2019). A methodological approach on the procedural effectiveness of EIA: the case of Sardinia. City Territ Archit, 6, 1. https://doi.org/10.1186/s40410-019-0100-5

Chanchitpricha, C. \& Bond, A. (2013). Conceptualising the effectiveness of impact assessment processes. Environmental Impact Assessment Review, 43, 65-72. https://doi.org/10.1016/j.eiar.2013.05.006

Comisión para el Desarrollo Minero Sostenible. (2020). Informe Final Propuestas de Medidas Normativas, de Gestión y de Política Pública para afianzar la Sostenibilidad del Sector Minero. Lima, Perú: Comisión para el Desarrollo Minero Sostenible.

Conesa, V. (2010). Guía metodológica para la evaluación del impacto ambiental. Madrid, España: Mundi-Prensa.

Decreto Legislativo $N^{\circ}$ 613. Código del Medio Ambiente y Recursos Naturales. Diario Oficial El Peruano, Lima, Perú, 08 de setiembre de 1990.

Decreto Legislativo $\mathrm{N}^{\circ} 757$. Ley marco para el crecimiento de la inversión privada. Diario Oficial El Peruano, Lima, Perú, 13 de noviembre de 1991. 
DIMENSIONES CONCEPTUALES PARA EVALUAR LA EFECTIVIDAD DE LA EVALUACIÓN DEL IMPACTO AMBIENTAL, BAJO LA LEGISLACIÓN AMBIENTAL PERUANA

Decreto Legislativo $\mathrm{N}^{\circ}$ 1500. Decreto Legislativo que establece Medidas Especiales para Reactivar, Mejorar y Optimizar la Ejecución de los Proyectos de Inversión Pública, Privada y Público Privada ante el Impacto del Covid-19. Diario Oficial El Peruano, Lima, Perú, 11 de mayo de 2020.

Decreto Supremo Nº19-2009-MINAM. Aprueban el Reglamento de la Ley № 27446, Ley del Sistema Nacional de Evaluación de Impacto Ambiental. Diario Oficial El Peruano, Lima, Perú, 25 de setiembre de 2009.

Decreto Supremo N 004-2013-PCM. Aprueba la Política Nacional de Modernización de la Gestión Pública. Diario Oficial El Peruano, Lima, Perú, 09 de enero de 2013.

Decreto Supremo N $^{\circ}$ 237-2019-EF. Aprueban el Plan Nacional de Competitividad y Productividad. Diario Oficial El Peruano, Lima, Perú, 28 de julio de 2019.

Decreto Supremo $N^{\circ}$ 023-2021-MINAM. Decreto Supremo que aprueba la Política Nacional del Ambiente al 2030. Diario Oficial El Peruano, Lima, Perú, 25 de julio de 2021.

Defensoría del Pueblo (2020). Informe de Adjuntía № 03-2020-DP/AMASPPI.MA, Participación ciudadana ambiental en tiempos de COVID-19. Recomendaciones para garantizar la protección de derechos en la evaluación del impacto ambiental de proyectos de inversión. Lima, Perú: Adjuntía del Medio Ambiente, Servicios Públicos y Pueblos Indígenas de la Defensoría del Pueblo. Espinoza, D. (2020). La Evaluación del Impacto Ambiental y la Calidad del Servicio de Certificación Ambiental para los Proyectos Energéticos en el Perú. Tesis para optar al grado académico de maestro en gestión pública con mención en sistemas energéticos. Centro de Altos Estudios Nacionales. Perú.

Günther, M.; Geibler, G. y Köppel, J. (2017). Many roads may lead to Rome: Selected features of quality control within environmental assessment systems in the US, NL, CA, and UK. Environmental Impact Assessment Review, 62, 250-258.

International Association for Impact Assessment (IAIA) (2020). Re-Envisioning Impact Assessment. Fargo, Estados Unidos. Recuperado: https://www.iaia.org news-details.php?ID=124

Jha-Thakur, U.; Gazzola, P.; Peel, D.; Fischer, T.B.; Kidd, S. (2009). Effectiveness of strategic environmental assessment - the significance of learning. Impact Assessment and Project Appraisal, 27(2). 133-144.

Khan, M.; Chaudhry, M. N.; Ahmad, S. R.; Mehmood, S. y Saif, A. (2020). Performance of EIA authority and effectiveness of EIA system in Pakistan. Environmental Impact Assessment Review, 81. https://doi.org/10.1016/j.eiar.2019.106357

Kolhoff, A. J.; Runhaar Hens, A.C.; Gugushvili, T.; Sonderegger, G.; Van der Leest, B. y Driessen, P.P.J. (2016). The influence of actor capacities on EIA system performance in low and middle income countries -Cases from Georgia and Ghana. Environmental Impact Assessment Review, 57, 167-177. https://doi.org/10.1016/j.eiar.2015.11.011

Ley $N^{\circ}$ 27446. Ley del Sistema Nacional de Evaluación del Impacto Ambiental. Diario Oficial El Peruano, Lima, Perú, 23 de abril de 2001. 
Ministerio del Ambiente (2021). Orientaciones para la aplicación en el Perú de la Evaluación Ambiental Estratégica con pensamiento estratégico. Viceministerio de Gestión Ambiental. Lima, Perú.

Nilsson, M. (2005). Learning frames and environmental policy integration: the case of Swedish energy policy. Environment and Planning C, 23(2), 207-226.

Nita, A. (2019). Empowering impact assessments knowledge and international research collaboration - A bibliometric analysis of Environmental Impact Assessment Review journal. Environmental Impact Assessment Review, 78. https://doi.org/10.1016/j.eiar.2019.106283

Pope, J.; Annandale, D.; Morrison-Saunders, A. (2004). Conceptualising sustainability assessment. Environmental Impact Assessment Review ,24(6), 595-616.

Pope, J.; Bond, A.; Cameron, C.; Retief, F.; Morrison-Saunders, A. (2018). Are current effectiveness criteria fit for purpose? Using a controversial strategic assessment as a test case. Environmental Impact Assessment Review, 70, 34-44. https://doi.org/10.1016/j.eiar.2018.01.004

Ramos, C. (2007). Cómo Hacer Una Tesis de Derecho y No Envejecer en el Intento. Lima, Perú: Editora Grijley.

Sadler, B. (1996). Environmental Assessment in a Changing World: Evaluating Practice to Improve Performance. Final Report of the International Study of the Effectiveness of Environmental Assessment. Ottawa, Canadian Environmental Assessment Agency and International Association for Impact Assessment.

Sánchez E. \& Falero M. (2015). ¿A qué paradigma de gestión de la naturaleza adscribe lanormativa peruanasobre calidad ambiental? EcologíaAplicada,14(2),163-174.

Shakil, S. H. \& Ananya, T. H. (2015). Effectiveness of Environmental Impact Assessment (EIA): Bangladesh Perspective. Bangladesh e-Journal of Sociology, 12(1), 115125. https://doi.org/10.2139/ssrn.2381707

The Institute of Environmental Management and Assessment (IEMA) (2020). Digital Impact Assessment: A Primer for Embracing Innovation and Digital Working. Lincoln, Reino Unido: IEMA.

Theophilou, V.; Bond, A. \& Cashmore, M. (2010). Application of the SEA Directive to EU structural funds: Perspectives on effectiveness. Environmental Impact Assessment Review, 30(2), 136-144. https://doi.org/10.1016/j.eiar. 2009.08.001

Zhang, Jie; Kørnøv, Lone; Christensen, Per (2020). A historical review of the cumulative science in SEA effectiveness. Environmental Impact Assessment Review, 83. https://doi.org/10.1016/j.eiar.2020.106412 